\title{
On sequences of large homoclinic solutions for a difference equations on the integers
}

\section{Robert Stegliński*}

\section{"Correspondence:}

robert.steglinski@p.lodz.pl Institute of Mathematics, Technical University of Lodz, Wolczanska 215, Lodz, 90-924, Poland

\section{Springer}

\begin{abstract}
In this paper, we determine a concrete interval of positive parameters $\lambda$, for which we prove the existence of infinitely many homoclinic solutions for a discrete problem

$$
-\Delta \phi_{p}(\Delta u(k-1))+a(k) \phi_{p}(u(k))=\lambda f(k, u(k)), \quad k \in \mathbb{Z},
$$

where the nonlinear term $f: \mathbb{Z} \times \mathbb{R} \rightarrow \mathbb{R}$ has an appropriate behavior at infinity, without any symmetry assumptions. The approach is based on critical point theory.

MSC: 39A10; 47J30; 35B38

Keywords: difference equations; discrete $p$-Laplacian; variational methods; infinitely many solutions
\end{abstract}

\section{Introduction}

In the present paper we deal with the following nonlinear second-order difference equation:

$$
\begin{cases}-\Delta \phi_{p}(\Delta u(k-1))+a(k) \phi_{p}(u(k))=\lambda f(k, u(k)) & \text { for all } k \in \mathbb{Z} \\ u(k) \rightarrow 0 & \text { as }|k| \rightarrow \infty\end{cases}
$$

Here $p>1$ is a real number, $\lambda$ is a positive real parameter, $\phi_{p}(t)=|t|^{p-2} t$ for all $t \in \mathbb{R}$, $a: \mathbb{Z} \rightarrow \mathbb{R}$ is a positive weight function, while $f: \mathbb{Z} \times \mathbb{R} \rightarrow \mathbb{R}$ is a continuous function. Moreover, the forward difference operator is defined as $\Delta u(k-1)=u(k)-u(k-1)$. We say that a solution $u=\{u(k)\}$ of (1) is homoclinic if $\lim _{|k| \rightarrow \infty} u(k)=0$.

Difference equations represent the discrete counterpart of ordinary differential equations and are usually studies in connection with numerical analysis. We may regard (1) as being a discrete analog of the following second-order differential equation:

$$
-\left(\phi_{p}\left(x^{\prime}(t)\right)\right)^{\prime}+a(t) \phi_{p}(x(t))=f(t, x(t)), \quad t \in \mathbb{R} .
$$

However, the relations between discretization and its continuous counterpart are not as direct as it may seem; see for example [1]. The case $p=2$ in (1) has been motivated in part

(c) 2016 Stegliński. This article is distributed under the terms of the Creative Commons Attribution 4.0 International License (http://creativecommons.org/licenses/by/4.0/), which permits unrestricted use, distribution, and reproduction in any medium, provided you give appropriate credit to the original author(s) and the source, provide a link to the Creative Commons license, and indicate if changes were made. 
by searching standing waves for the nonlinear Schrodinger equation

$$
i \dot{\psi}_{k}+\Delta^{2} \psi_{k}-v_{k} \psi_{k}+f\left(k, \psi_{k}\right)=0, \quad k \in \mathbb{Z}
$$

(for details see $[2,3]$ ).

Most of the classical methods used in the case of differential equations lead to existence results for the solutions of a difference equation. Variational methods for difference equations consist in seeking solutions as critical points for a suitable associated energy functional defined on a convenient Banach space. In the first approaches to the issue, the variational methods are applied to boundary value problems on bounded discrete intervals, which leads to the study of an energy functional defined on a finite-dimensional Banach space (see [4-9]). In the case of difference equations on unbounded discrete intervals (typically, on the whole set of integers $\mathbb{Z}$ ) solutions are sought in a subspace of the sequence space $l^{p}$ which is still infinite-dimensional but compactly embedded into $l^{p}$ (see [10-12]).

Our idea here is to investigate the existence of infinitely many solutions to problem (1) by using a critical point theorem obtained in [13]; see Theorem 2 . This theorem was used in a continuous case in [14] and in discrete but finite-dimensional analog of problem (1) in [15-17]. In analogy with the cited papers, in our approach we do not require any symmetry hypothesis.

A special case of our contributions reads as follows. For $a: \mathbb{Z} \rightarrow \mathbb{R}$ and the continuous mapping $f: \mathbb{Z} \times \mathbb{R} \rightarrow \mathbb{R}$ define the following conditions:

(A) $a(k) \geq \alpha>0$ for all $k \in \mathbb{Z}, a(k) \rightarrow+\infty$ as $|k| \rightarrow+\infty$;

(F) $\lim _{t \rightarrow 0} \frac{f(k, t)}{t^{p-1}}=0$ uniformly for all $k \in \mathbb{Z}$;

$\left(\mathrm{F}_{2}\right) \liminf _{t \rightarrow+\infty} \frac{\sum_{k \in \mathbb{Z}} \max _{|\xi| \leq t} F(k, \xi)}{t^{p}}=0$;

$\left(\mathrm{F}_{3}\right) \lim \sup _{(k, t) \rightarrow(+\infty,+\infty)} \frac{F(k, t)}{(2+a(k))|t|^{p}}=+\infty$;

$\left(\mathrm{F}_{4}\right) \sup _{k \in \mathbb{Z}}\left(\lim \sup _{t \rightarrow+\infty} \frac{F(k, t)}{(2+a(k))|t|^{p}}\right)=+\infty$,

where $F(k, t)$ is the primitive function of $f(k, t)$, that is, $F(k, t)=\int_{0}^{t} f(k, s) d s$ for every $t \in \mathbb{R}$ and $k \in \mathbb{Z}$. The solutions are found in the normed space $(X,\|\cdot\|)$, where $X=\{u: \mathbb{Z} \rightarrow \mathbb{R}$ : $\left.\sum_{k \in \mathbb{Z}} a(k)|u(k)|^{p}<\infty\right\}$ and $\|u\|=\left[\sum_{k \in \mathbb{Z}} a(k)|u(k)|^{p}\right]^{\frac{1}{p}}$.

Theorem 1 Assume that $(\mathrm{A}),\left(\mathrm{F}_{1}\right)$, and $\left(\mathrm{F}_{2}\right)$ are satisfied. Moreover, assume that either $\left(F_{3}\right)$ or $\left(F_{4}\right)$ is satisfied. Then for each $\lambda>0$, the problem (1) admits a sequence of solutions in $X$ whose norms tend to infinity.

The plan of the paper is the following: Section 2 is devoted to our abstract framework, while Section 3 is dedicated to the main results. Finally, we give two examples which illustrate the independence of conditions $\left(\mathrm{F}_{3}\right)$ and $\left(\mathrm{F}_{4}\right)$ and a third example which corresponds to the general Theorem 6 .

\section{Abstract framework}

Our main tool is a general critical points theorem due to Bonanno and Molica Bisci (see [18]), which is a generalization of result of Ricceri [13]. Here we state it in a smooth version for the reader's convenience. 
Theorem 2 Let $(E,\|\cdot\|)$ be a reflexive real Banach space, let $\Phi, \Psi: E \rightarrow \mathbb{R}$ be two continuously differentiable functionals with $\Phi$ coercive, i.e. $\lim _{\|u\| \rightarrow \infty} \Phi(u)=+\infty$, and a sequentially weakly lower semicontinuous functional and $\Psi$ a sequentially weakly upper semicontinuous functional. For every $r>\inf _{E} \Phi$, let us put

$$
\varphi(r):=\inf _{u \in \Phi^{-1}((-\infty, r))} \frac{\left(\sup _{v \in \Phi^{-1}((-\infty, r))} \Psi(v)\right)-\Psi(u)}{r-\Phi(u)}
$$

and

$$
\gamma:=\liminf _{r \rightarrow+\infty} \varphi(r)
$$

Let $J_{\lambda}:=\Phi(u)-\lambda \Psi(u)$ for all $u \in E$. If $\gamma<+\infty$ then, for each $\lambda \in\left(0, \frac{1}{\gamma}\right)$, the following alternative holds:

$$
\text { either }
$$

(a) $J_{\lambda}$ possesses a global minimum,

$$
\text { or }
$$

(b) there is a sequence $\left\{u_{n}\right\}$ of critical points (local minima) of $J_{\lambda}$ such that $\lim _{n \rightarrow+\infty} \Phi\left(u_{n}\right)=+\infty$.

We begin by defining some Banach spaces. For all $1 \leq p<+\infty$, we denote $\ell^{p}$ the set of all functions $u: \mathbb{Z} \rightarrow \mathbb{R}$ such that

$$
\|u\|_{p}^{p}=\sum_{k \in \mathbb{Z}}|u(k)|^{p}<+\infty .
$$

Moreover, we denote by $\ell^{\infty}$ the set of all functions $u: \mathbb{Z} \rightarrow \mathbb{R}$ such that

$$
\|u\|_{\infty}=\sup _{k \in \mathbb{Z}}|u(k)|<+\infty .
$$

We set

$$
X=\left\{u: \mathbb{Z} \rightarrow \mathbb{R}: \sum_{k \in \mathbb{Z}} a(k)|u(k)|^{p}<\infty\right\}
$$

and

$$
\|u\|=\left[\sum_{k \in \mathbb{Z}} a(k)|u(k)|^{p}\right]^{\frac{1}{p}} .
$$

Clearly we have

$$
\|u\|_{\infty} \leq\|u\|_{p} \leq \alpha^{-\frac{1}{p}}\|u\| \quad \text { for all } u \in X .
$$

As is shown in [11], Proposition $3,(X,\|\cdot\|)$ is a reflexive Banach space and the embedding $X \hookrightarrow l^{p}$ is compact.

Let

$$
\Phi(u):=\frac{1}{p} \sum_{k \in \mathbb{Z}}\left[|\Delta u(k-1)|^{p}+a(k)|u(k)|^{p}\right] \quad \text { for all } u \in X
$$


and

$$
\Psi(u):=\sum_{k \in \mathbb{Z}} F(k, u(k)) \quad \text { for all } u \in l^{p}
$$

where $F(k, s)=\int_{0}^{s} f(k, t) d t$ for $s \in \mathbb{R}$ and $k \in \mathbb{Z}$. Let $J_{\lambda}: X \rightarrow \mathbb{R}$ be the functional associated to problem (1) defined by

$$
J_{\lambda}(u)=\Phi(u)-\lambda \Psi(u) .
$$

Proposition 3 Assume that $(\mathrm{A})$ and $\left(\mathrm{F}_{1}\right)$ are satisfied. Then

(a) $\Phi \in C^{1}(X)$;

(b) $\Psi \in C^{1}\left(l^{p}\right)$ and $\Psi \in C^{1}(X)$;

(c) $J_{\lambda} \in C^{1}(X)$ and for all $\lambda>0$ every critical point $u \in X$ of $J_{\lambda}$ is a homoclinic solution of problem (1).

For a proof see Propositions 5, 6, and 7 in [11].

Proposition 4 Assume that $(\mathrm{A})$ and $\left(\mathrm{F}_{1}\right)$ are satisfied. Then $\Phi$ is coercive and sequentially weakly lower semicontinuous functional and $\Psi$ is sequentially weakly upper semicontinuous functional.

Proof We have

$$
\Phi(u) \geq \frac{1}{p}\|u\|^{p} \rightarrow+\infty,
$$

as $\|u\| \rightarrow+\infty$, and so $\Phi$ is coercive. Now, let $u_{m} \rightarrow u$ weakly in $X$. By the compactness of the embedding $X \hookrightarrow l^{p}$, we may assume that $u_{m} \rightarrow u$ in $l^{p}$. The rest of the proposition follows from the fact that the norm $\|\cdot\|$ in $X$ is sequentially weakly lower semicontinuous functional, the functional $\Psi$ is continuous on $l^{p}$, and the functional $l^{p} \ni u \mapsto$ $\frac{1}{p} \sum_{k \in \mathbb{Z}}|\Delta u(k-1)|^{p} \in \mathbb{R}$ is continuous on $l^{p}$.

Proposition 5 Let $\left\{u_{m}\right\}$ be a sequence in $X$ such that $\Phi\left(u_{m}\right) \rightarrow+\infty$. Then $\left\|u_{m}\right\| \rightarrow+\infty$.

Proof By the classical Minkowski inequality

$$
\left(\sum_{i=1}^{n}\left|x_{i}+y_{i}\right|^{p}\right)^{\frac{1}{p}} \leq\left(\sum_{i=1}^{n}\left|x_{i}\right|^{p}\right)^{\frac{1}{p}}+\left(\sum_{i=1}^{n}\left|y_{i}\right|^{p}\right)^{\frac{1}{p}}
$$

for all $n \in \mathbb{N}, x_{1}, \ldots, x_{n}, y_{1}, \ldots, y_{n} \in \mathbb{R}$, we have

$$
\left(\sum_{|k| \leq h}|\Delta u(k-1)|^{p}\right)^{\frac{1}{p}} \leq\left(\sum_{|k| \leq h}|u(k-1)|^{p}\right)^{\frac{1}{p}}+\left(\sum_{|k| \leq h}|u(k)|^{p}\right)^{\frac{1}{p}}
$$

for all $h \in \mathbb{N}$ and $u \in X$. Letting $h \rightarrow+\infty$ we obtain

$$
\left(\sum_{k \in \mathbb{Z}}|\Delta u(k-1)|^{p}\right)^{\frac{1}{p}} \leq 2\|u\|_{p}
$$


From this and inequality (2) we conclude

$$
\begin{aligned}
\Phi(u) & =\frac{1}{p} \sum_{k \in \mathbb{Z}}\left[|\Delta u(k-1)|^{p}+a(k)|u(k)|^{p}\right] \\
& \leq \frac{1}{p}\left(2\|u\|_{p}^{p}+\|u\|^{p}\right) \leq \frac{2+\alpha}{p \alpha}\|u\|^{p},
\end{aligned}
$$

which proves the proposition.

\section{Main theorem}

Now we will formulate and prove a stronger form of Theorem 1 . Let

$$
\begin{aligned}
& A:=\liminf _{t \rightarrow+\infty} \frac{\sum_{k \in \mathbb{Z}} \max _{|\xi| \leq t} F(k, \xi)}{t^{p}} \\
& B_{ \pm, \pm}:=\limsup _{(k, t) \rightarrow( \pm \infty, \pm \infty)} \frac{F(k, t)}{(2+a(k))|t|^{p}}
\end{aligned}
$$

and

$$
B_{ \pm}:=\sup _{k \in \mathbb{Z}}\left(\limsup _{t \rightarrow \pm \infty} \frac{F(k, t)}{(2+a(k))|t|^{p}}\right) .
$$

Set $B:=\max \left\{B_{ \pm, \pm}, B_{ \pm}\right\}$. For convenience we put $\frac{1}{0^{+}}=+\infty$ and $\frac{1}{+\infty}=0$.

Theorem 6 Assume that $(\mathrm{A})$ and $\left(\mathrm{F}_{1}\right)$ are satisfied and assume that the following inequality holds: $A<\alpha \cdot B$. Then, for each $\lambda \in\left(\frac{1}{B p}, \frac{\alpha}{A p}\right)$, problem (1) admits a sequence of solutions in $X$ whose norms tend to infinity.

Proof It is clear that $A \geq 0$. Put $\lambda \in\left(\frac{1}{B p}, \frac{\alpha}{A p}\right)$ and put $\Phi, \Psi, J_{\lambda}$ as in the previous section. Our aim is to apply Theorem 2 to the function $J_{\lambda}$. By Lemma 4 , the functional $\Phi$ is the locally Lipschitz, coercive and sequentially weakly lower semicontinuous functional and $\Psi$ is the locally Lipschitz, sequentially weakly upper semicontinuous functional. We will show that $\gamma<+\infty$. Let $\left\{c_{m}\right\} \subset(0,+\infty)$ be a sequence such that $\lim _{m \rightarrow \infty} c_{m}=+\infty$ and

$$
\lim _{m \rightarrow+\infty} \frac{\sum_{k \in \mathbb{Z}} \max _{|\xi| \leq c_{m}} F(k, \xi)}{c_{m}^{p}}=A .
$$

Set

$$
r_{m}:=\frac{\alpha}{p} c_{m}^{p}
$$

for every $m \in \mathbb{N}$. Then, if $v \in X$ and $\Phi(v)<r_{m}$, one has

$$
\|v\|_{\infty} \leq \alpha^{-\frac{1}{p}}\|v\| \leq \alpha^{-\frac{1}{p}}(p \Phi(v))^{\frac{1}{p}}<c_{m}
$$

which gives

$$
\Phi^{-1}\left(\left(-\infty, r_{m}\right)\right) \subset\left\{v \in X:\|v\|_{\infty} \leq c_{m}\right\} .
$$


From this and $\Phi(0)=\Psi(0)=0$ we have

$$
\begin{aligned}
\varphi\left(r_{m}\right) & \leq \frac{\sup _{\Phi(v)<r_{m}} \sum_{k \in \mathbb{Z}} F(k, v(k))}{r_{m}} \leq \frac{\sum_{k \in \mathbb{Z}} \max _{|t| \leq c_{m}} F(k, t)}{r_{m}} \\
& =\frac{p}{\alpha} \cdot \frac{\sum_{k=1}^{T} \max _{|t| \leq c_{m}} F(k, t)}{c_{m}^{p}}
\end{aligned}
$$

for every $m \in \mathbb{N}$. This gives

$$
\gamma \leq \lim _{m \rightarrow+\infty} \varphi\left(r_{m}\right) \leq \frac{p}{\alpha} \cdot A<\frac{1}{\lambda}<+\infty .
$$

Now, our conclusion follows provided that $J_{\lambda}$ does not possess a global minimum.

Case 1. First assume that $B=\max \left\{B_{ \pm, \pm}\right\}$. Without loss of generality we can assume that $B=B_{+,+}$. We begin with $B=+\infty$. Accordingly, let $M$ be such that $M>\frac{1}{\lambda p}$. There exist a sequence of positive integers $\left\{k_{m}\right\}$ and a sequence of real numbers $\left\{b_{m}\right\}$ with $b_{m} \geq 1$ and $\lim _{m \rightarrow+\infty} b_{m}=+\infty$ and such that

$$
F\left(k_{m}, b_{m}\right)>M\left(2+a\left(k_{m}\right)\right) b_{m}^{p}
$$

for all $m \in \mathbb{N}$. Thus, take in $X$ a sequence $\left\{s_{m}\right\}$ such that, for every $m \in \mathbb{N}, s_{m}\left(k_{m}\right)=b_{m}$, and $s_{m}(k)=0$ for every $k \in \mathbb{Z} \backslash\left\{k_{m}\right\}$. Then one has

$$
\begin{aligned}
J_{\lambda}\left(s_{m}\right) & =\frac{1}{p} \sum_{k \in \mathbb{Z}}\left(\left|\Delta s_{m}(k-1)\right|^{p}+a(k)\left|s_{m}(k)\right|^{p}\right)-\lambda \sum_{k \in \mathbb{Z}} F\left(k, s_{m}(k)\right) \\
& <\frac{2}{p} b_{m}^{p}+\frac{1}{p} a\left(k_{m}\right) b_{m}^{p}-\lambda M\left(2+a\left(k_{m}\right)\right) b_{m}^{p} \\
& =\left(\frac{1}{p}-\lambda M\right)\left(2+a\left(k_{m}\right)\right) b_{m}^{p},
\end{aligned}
$$

which gives $\lim _{m \rightarrow+\infty} J_{\lambda}\left(s_{m}\right)=-\infty$.

Next, assume that $B<+\infty$. Since $\lambda>\frac{1}{B p}$, we can fix $\varepsilon>0$ such that $\varepsilon<B-\frac{1}{\lambda p}$. Therefore, also taking $\left\{k_{m}\right\}$, a sequence of positive integers, and $\left\{b_{m}\right\}$, a sequence of real numbers with $b_{m} \geq 1$ such that $\lim _{m \rightarrow+\infty} b_{m}=+\infty$ and

$$
(B-\varepsilon)\left(2+a\left(k_{m}\right)\right) b_{m}^{p}<F\left(k_{m}, b_{m}\right)<(B+\varepsilon)\left(2+a\left(k_{m}\right)\right) b_{m}^{p}
$$

for all $m \in \mathbb{N}$, choosing $\left\{s_{m}\right\}$ in $X$ as above, one has

$$
J_{\lambda}\left(s_{m}\right)<\left(\frac{1}{p}-\lambda(B-\varepsilon)\right)\left(2+a\left(k_{m}\right)\right) b_{m}^{p} .
$$

So, also in this case, $\lim _{m \rightarrow+\infty} J_{\lambda}\left(s_{m}\right)=-\infty$.

Case 2. Now assume that $B=\max \left\{B_{ \pm}\right\}$. Without loss of generality we can assume that $B=B_{+}$. We begin with $B=+\infty$. Accordingly, let $M$ be such that $M>\frac{1}{\lambda p}$. There exists an index $k_{0} \in \mathbb{Z}$ such that

$$
\limsup _{t \rightarrow+\infty} \frac{F\left(k_{0}, t\right)}{\left(2+a\left(k_{0}\right)\right)|t|^{p}}>M
$$


Then there exists a sequence of real numbers $\left\{b_{m}\right\}$ with $b_{m} \geq 1$ such that $\lim _{m \rightarrow+\infty} b_{m}=$ $+\infty$ and

$$
F\left(k_{0}, b_{m}\right)>M\left(2+a\left(k_{0}\right)\right) b_{m}^{p}
$$

for all $m \in \mathbb{N}$. Thus, take in $X$ a sequence $\left\{s_{m}\right\}$ such that, for every $m \in \mathbb{N}, s_{m}\left(k_{0}\right)=b_{m}$, and $s_{m}(k)=0$ for every $k \in \mathbb{Z} \backslash\left\{k_{0}\right\}$. Then one has

$$
\begin{aligned}
J_{\lambda}\left(s_{m}\right) & =\frac{1}{p} \sum_{k \in \mathbb{Z}}\left(\left|\Delta s_{m}(k-1)\right|^{p}+a(k)\left|s_{m}(k)\right|^{p}\right)-\lambda \sum_{k \in \mathbb{Z}} F\left(k, s_{m}(k)\right) \\
& <\frac{2}{p} b_{m}^{p}+\frac{1}{p} a\left(k_{0}\right) b_{m}^{p}-\lambda M\left(2+a\left(k_{0}\right)\right) b_{m}^{p} \\
& =\left(\frac{1}{p}-\lambda M\right)\left(2+a\left(k_{0}\right)\right) b_{m}^{p}
\end{aligned}
$$

which gives $\lim _{m \rightarrow+\infty} J_{\lambda}\left(s_{m}\right)=-\infty$.

Next, assume that $B<+\infty$. Since $\lambda>\frac{1}{B p}$, we can fix $\varepsilon>0$ such that $\varepsilon<B-\frac{1}{\lambda p}$. Therefore, there exists an index $k_{0} \in \mathbb{Z}$ such that

$$
\limsup _{t \rightarrow+\infty} \frac{F\left(k_{0}, t\right)}{\left(2+a\left(k_{0}\right)\right)|t|^{p}}>B-\varepsilon
$$

and taking $\left\{b_{m}\right\}$, a sequence of real numbers with $b_{m} \geq 1$ such that $\lim _{m \rightarrow+\infty} b_{m}=+\infty$ and

$$
F\left(k_{0}, b_{m}\right)>(B-\varepsilon)\left(2+a\left(k_{0}\right)\right) b_{m}^{p}
$$

for all $m \in \mathbb{N}$, choosing $\left\{s_{m}\right\}$ in $X$ as above, one has

$$
J_{\lambda}\left(s_{m}\right)<\left(\frac{1}{p}-\lambda(B-\varepsilon)\right)\left(2+a\left(k_{0}\right)\right) b_{m}^{p}
$$

So, also in this case, $\lim _{m \rightarrow+\infty} J_{\lambda}\left(s_{m}\right)=-\infty$. Finally, the above facts mean that $J_{\lambda}$ does not possesses a global minimum. Hence, by Theorem 2, we obtain a sequence $\left\{u_{m}\right\}$ of critical points (local minima) of $J_{\lambda}$ such that $\lim _{m \rightarrow+\infty} \Phi\left(u_{m}\right)=+\infty$. Proposition 5 now implies $\lim _{m \rightarrow+\infty}\left\|u_{m}\right\|=+\infty$. The proof is complete.

Remark 1 Theorem 1 follows from Theorem 6 since the condition $\left(\mathrm{F}_{3}\right)$ means that $B_{+,+}=$ $+\infty$ and the condition $\left(\mathrm{F}_{4}\right)$ means that $B_{+}=+\infty$, so $B=+\infty$. Moreover, $\left(\mathrm{F}_{2}\right)$ implies $A=0$. Hence, we obtain the interval of all positive parameters, for which the treated problem admits infinitely many solutions whose norms tend to infinity.

Remark 2 If we replace the condition $\left(\mathrm{F}_{1}\right)$ by

$\left(\mathrm{F}_{1}^{+}\right) \lim _{t \rightarrow 0^{+}} \frac{f(k, t)}{t^{p-1}}=0$ uniformly for all $k \in \mathbb{Z}$

in Theorem 1, then we can obtain the sequence of positive solutions of the problem (1). It can be proved by using Lemmas 5 and 6 in [10].

\section{Examples}

Now, we will show the example of a function for which we can apply Theorem 1. First we give an example of a function $f$ for which $\left(\mathrm{F}_{3}\right)$ arises, but $\left(\mathrm{F}_{4}\right)$ is not satisfied. 
Example 1 Let $\{a(k)\}$ be a sequence of positive numbers. Put $b_{1}=1$. Let $\left\{b_{m}\right\},\left\{c_{m}\right\},\left\{h_{m}\right\}$ be sequences defined recursively by

$$
\begin{cases}b_{m+1}=\left[m(m+1)(2+a(m)) c_{m}^{p}+\frac{m+1}{m} b_{m}^{p}\right]^{\frac{1}{p}} & \text { for } m \geq 1 \\ c_{m}=1+b_{m} & \text { for } m \geq 1 \\ h_{m}=\frac{1}{m+1} b_{m+1}^{p}-\frac{1}{m} b_{m}^{p} & \text { for } m \geq 1\end{cases}
$$

It is easy to see that $b_{m}<c_{m}<b_{m+1}$ for every $m \in \mathbb{N}$ and $\lim _{m \rightarrow+\infty} b_{m}=\lim _{m \rightarrow+\infty} c_{m}=$ $+\infty$. For every nonpositive integer $k$ let $f(k, \cdot): \mathbb{R} \rightarrow \mathbb{R}$ be identically the zero function. For every positive integer $k$ let $f(k, \cdot): \mathbb{R} \rightarrow \mathbb{R}$ be any nonnegative continuous function such that $f(k, t)=0$ for $t \in \mathbb{R} \backslash\left(b_{k}, c_{k}\right)$ and $\int_{b_{k}}^{c_{k}} f(k, t) d t=h_{k}$. The condition $\left(\mathrm{F}_{1}\right)$ is now obviously satisfied.

Set $F(k, t):=\int_{0}^{t} f(k, s) d s$ for every $t \in \mathbb{R}$ and $k \in \mathbb{Z}$. Then

$$
\begin{aligned}
\liminf _{t \rightarrow+\infty} \frac{\sum_{k \in \mathbb{Z}} \max _{|\xi| \leq t} F(k, \xi)}{t^{p}} & \leq \lim _{m \rightarrow+\infty} \frac{\sum_{k \in \mathbb{Z}} \max _{|\xi| \leq b_{m}} F(k, \xi)}{b_{m}^{p}} \\
& =\lim _{m \rightarrow+\infty} \frac{\sum_{k=1}^{m-1} F\left(k, c_{k}\right)}{b_{m}^{p}} \\
& =\lim _{m \rightarrow+\infty} \frac{\sum_{k=1}^{m-1} h_{k}}{b_{m}^{p}} \\
& =\lim _{m \rightarrow+\infty} \frac{\frac{1}{m} b_{m}^{p}-1}{b_{m}^{p}}=0
\end{aligned}
$$

and

$$
\begin{aligned}
\limsup _{(k, t) \rightarrow(+\infty,+\infty)} \frac{F(k, t)}{(2+a(k))|t|^{p}} & \geq \lim _{m \rightarrow+\infty} \frac{F\left(m, c_{m}\right)}{(2+a(m)) c_{m}^{p}} \\
& =\lim _{m \rightarrow+\infty} \frac{h_{m}}{(2+a(m)) c_{m}^{p}} \\
& =\lim _{m \rightarrow+\infty} \frac{m(2+a(m)) c_{m}^{p}}{(2+a(m)) c_{m}^{p}}=+\infty
\end{aligned}
$$

Moreover,

$$
\sup _{k \in \mathbb{Z}}\left(\limsup _{t \rightarrow+\infty} \frac{F(k, t)}{(2+a(k))|t|^{p}}\right)=0
$$

Now we give an example of a function $f$ for which $\left(\mathrm{F}_{4}\right)$ arises, but $\left(\mathrm{F}_{3}\right)$ is not satisfied.

Example 2 Let $\{a(k)\}$ be a sequence of positive numbers. Put $b_{1}=1$. Let $\left\{b_{m}\right\},\left\{c_{m}\right\},\left\{h_{m}\right\}$ be sequences defined recursively by

$$
\begin{cases}b_{m}=\left(m \sum_{k=1}^{m-1} h_{k}\right)^{\frac{1}{p}} & \text { for } m \geq 2, \\ c_{m}=1+b_{m} & \text { for } m \geq 1 \\ h_{m}=m(2+a(0)) c_{m}^{p}-\frac{1}{m} b_{m}^{p} & \text { for } m \geq 1 .\end{cases}
$$


It is easy to see that $b_{m}<c_{m}<b_{m+1}$ for every $m \in \mathbb{N}$ and $\lim _{m \rightarrow+\infty} b_{m}=\lim _{m \rightarrow+\infty} c_{m}=$ $+\infty$. Let $\tilde{g}: \mathbb{R} \rightarrow \mathbb{R}$ be the continuous nonnegative function given by

$$
\tilde{f}(s):=\sum_{m \in \mathbb{N}} 2 h_{m}\left(1-2\left|s-c_{m}+\frac{1}{2}\right|\right) \cdot \mathbf{1}_{\left[b_{m}, c_{m}\right]},
$$

where $\mathbf{1}_{[b, c]}$ is the indicator of the interval $[b, c]$. We check at once that, for every $m \in \mathbb{N}$,

$$
\int_{b_{m}}^{c_{m}} \tilde{f}(s) d s=h_{m}
$$

Set $f(0, s):=\tilde{f}(s)$ for $s \in \mathbb{R}$ and $f(k, s)=0$ for $k \in \mathbb{Z} \backslash\{0\}$ and $s \in \mathbb{R}$. Set $F(k, t):=\int_{0}^{t} f(k, s) d s$ for every $t \in \mathbb{R}$ and $k \in \mathbb{Z}$. Then $F\left(0, c_{m}\right)=\sum_{k=1}^{m} h_{k}$. The condition $\left(\mathrm{F}_{1}\right)$ is satisfied and

$$
\begin{aligned}
\liminf _{t \rightarrow+\infty} \frac{\sum_{k \in \mathbb{Z}} \max _{|\xi| \leq t} F(k, \xi)}{t^{p}} & \leq \lim _{m \rightarrow+\infty} \frac{\max _{|\xi| \leq b_{m}} F(0, \xi)}{b_{m}^{p}} \\
& =\lim _{m \rightarrow+\infty} \frac{F\left(0, c_{m-1}\right)}{b_{m}^{p}} \\
& =\lim _{m \rightarrow+\infty} \frac{\sum_{k=1}^{m-1} h_{k}}{m \sum_{k=1}^{m-1} h_{k}}=0
\end{aligned}
$$

and

$$
\begin{aligned}
\sup _{k \in \mathbb{Z}}\left(\limsup _{t \rightarrow+\infty} \frac{F(k, t)}{(2+a(k))|t|^{p}}\right) & =\limsup _{t \rightarrow+\infty} \frac{F(0, t)}{(2+a(0))|t|^{p}} \\
& \geq \lim _{m \rightarrow+\infty} \frac{F\left(0, c_{m}\right)}{(2+a(0)) c_{m}^{p}} \\
& =\lim _{m \rightarrow+\infty} \frac{\sum_{k=1}^{m} h_{k}}{\frac{1}{m}\left(\frac{1}{m} b_{m}^{p}+h_{m}\right)} \\
& =\lim _{m \rightarrow+\infty} \frac{m \sum_{k=1}^{m} h_{k}}{\sum_{k=1}^{m-1} h_{k}+h_{m}}=+\infty
\end{aligned}
$$

Moreover,

$$
\limsup _{(k, t) \rightarrow(+\infty,+\infty)} \frac{F(k, t)}{(2+a(k))|t|^{p}}=0
$$

Now, let $\{a(k)\}$ be a sequence of positive numbers such that $a(k) \rightarrow+\infty$ as $|k| \rightarrow+\infty$. Let $\alpha, \beta$ be any two strictly positive real numbers. We show that there is a continuous function $f: \mathbb{Z} \times \mathbb{R} \rightarrow \mathbb{R}$, such that the numbers $A$ and $B$, as defined above Theorem 6 , are equal to $\alpha$ and $\beta$, respectively. We can follow one of two strategies given by Example 1 and Example 2. We choose the first one.

Example 3 Let $m_{0} \in \mathbb{N}$ be such that $\alpha<(2+a(m)) \cdot \beta$ for every $m \geq m_{0}$. Put $b_{m_{0}}=1$. Let $\left\{b_{m}\right\},\left\{c_{m}\right\},\left\{h_{m}\right\}$ be sequences defined recursively by

$$
\begin{cases}b_{m+1}=\left[\frac{\beta}{\alpha}(2+a(m)) c_{m}^{p}+b_{m}^{p}\right]^{\frac{1}{p}} & \text { for } m \geq m_{0}, \\ c_{m}=1+b_{m} & \text { for } m \geq m_{0}, \\ h_{m}=\alpha\left(b_{m+1}^{p}-b_{m}^{p}\right) & \text { for } m \geq m_{0} .\end{cases}
$$


It is easy to see that $b_{m}<c_{m}<b_{m+1}$ for every $m \geq m_{0}$ and $\lim _{m \rightarrow+\infty} b_{m}=\lim _{m \rightarrow+\infty} c_{m}=$ $+\infty$. For every integer $k<m_{0}$ let $f(k, \cdot): \mathbb{R} \rightarrow \mathbb{R}$ be identically the zero function. For every integer $k \geq m_{0}$ let $f(k, \cdot): \mathbb{R} \rightarrow \mathbb{R}$ be any nonnegative continuous function such that $f(k, t)=0$ for $t \in \mathbb{R} \backslash\left(b_{k}, c_{k}\right)$ and $\int_{b_{k}}^{c_{k}} f(k, t) d t=h_{k}$. The condition $\left(\mathrm{F}_{1}\right)$ is now obviously satisfied.

Set $F(k, t):=\int_{0}^{t} f(k, s) d s$ for every $t \in \mathbb{R}$ and $k \in \mathbb{Z}$. Since

$$
\begin{aligned}
\frac{b_{m}^{p}}{c_{m}^{p}} \cdot \frac{\sum_{k \in \mathbb{Z}} \max _{|\xi| \leq b_{m}} F(k, \xi)}{b_{m}^{p}} & =\frac{\sum_{k \in \mathbb{Z}} \max _{|\xi| \leq c_{m-1}} F(k, \xi)}{c_{m}^{p}} \\
& \leq \inf _{t \in\left(c_{m-1}, c_{m}\right)} \frac{\sum_{k \in \mathbb{Z}} \max _{|\xi| \leq t} F(k, \xi)}{t^{p}} \\
& \leq \frac{\sum_{k \in \mathbb{Z}} \max _{|\xi| \leq b_{m}} F(k, \xi)}{b_{m}^{p}}
\end{aligned}
$$

is satisfied for all $m \geq m_{0}$, we have

$$
\liminf _{t \rightarrow+\infty} \frac{\sum_{k \in \mathbb{Z}} \max _{|\xi| \leq t} F(k, \xi)}{t^{p}}=\lim _{m \rightarrow+\infty} \frac{\sum_{k \in \mathbb{Z}} \max _{|\xi| \leq b_{m}} F(k, \xi)}{b_{m}^{p}}
$$

and since

$$
\frac{F\left(k, c_{k}\right)}{(2+a(k)) c_{k}^{p}} \leq \sup _{t \in \mathbb{R}} \frac{F(k, t)}{(2+a(k))|t|^{p}} \leq \frac{h_{k}}{(2+a(k)) b_{k}^{p}}=\frac{F\left(k, c_{k}\right)}{(2+a(k)) c_{k}^{p}} \cdot \frac{c_{k}^{p}}{b_{k}^{p}}
$$

is satisfied for all $k \geq m_{0}$, we have

$$
\limsup _{(k, t) \rightarrow(+\infty,+\infty)} \frac{F(k, t)}{(2+a(k))|t|^{p}}=\lim _{k \rightarrow+\infty} \frac{F\left(k, c_{k}\right)}{(2+a(k)) c_{k}^{p}} .
$$

In the same way as has been done in Example 1, we conclude that

$$
\liminf _{t \rightarrow+\infty} \frac{\sum_{k \in \mathbb{Z}} \max _{|\xi| \leq t} F(k, \xi)}{t^{p}}=\alpha
$$

and

$$
\limsup _{(k, t) \rightarrow(+\infty,+\infty)} \frac{F(k, t)}{(2+a(k))|t|^{p}}=\beta .
$$

Moreover,

$$
\sup _{k \in \mathbb{Z}}\left(\limsup _{t \rightarrow+\infty} \frac{F(k, t)}{(2+a(k))|t|^{p}}\right)=0
$$

From this we obtain $A=\alpha$ and $B=\beta$. 


\section{Acknowledgements}

The research was supported by Lodz University of Technology. The author would like to thank the referees for their valuable suggestions and comments.

Received: 18 November 2015 Accepted: 25 January 2016 Published online: 02 February 2016

\section{References}

1. Agarwal, RP: On multipoint boundary value problems for discrete equations. J. Math. Anal. Appl. 96(2), $520-534$ (1983)

2. Sun, G: On standing wave solutions for discrete nonlinear Schrodinger equations. Abstr. Appl. Anal. 2013, Article ID $436919(2013)$

3. Sun, G, Mai, A: Infinitely many homoclinic solutions for second order nonlinear difference equations with $p$-Laplacian Sci. World J. 2014, Article ID 276372 (2014)

4. Agarwal, RP, Perera, K, O'Regan, D: Multiple positive solutions of singular and nonsingular discrete problems via variational methods. Nonlinear Anal. 58, 69-73 (2004)

5. Cabada, A, lannizzotto, A, Tersian, S: Multiple solutions for discrete boundary value problems. J. Math. Anal. Appl. 356, 418-428 (2009)

6. Candito, P, Molica Bisci, G: Existence of two solutions for a second-order discrete boundary value problem. Adv. Nonlinear Stud. 11, 443-453 (2011)

7. Galewski, M, Głab, S: On the discrete boundary value problem for anisotropic equation. Electron. J. Math. Anal. Appl. $386,956-965(2012)$

8. Mihăilescu, M, Rădulescu, V, Tersian, S: Eigenvalue problems for anisotropic discrete boundary value problems. J. Differ. Equ. Appl. 15(6), 557-567 (2009)

9. Molica Bisci, G, Repovš, D: Existence of solutions for p-Laplacian discrete equations. Appl. Math. Comput. 242, 454-461 (2014)

10. Iannizzotto, A, Rădulescu, V: Positive homoclinic solutions for the discrete $p$-Laplacian with a coercive weight function. Differ. Integral Equ. 27(1-2), 35-44 (2014)

11. Iannizzotto, A, Tersian, S: Multiple homoclinic solutions for the discrete $p$-Laplacian via critical point theory. J. Math. Anal. Appl. 403, 173-182 (2013)

12. Ma, M, Guo, Z: Homoclinic orbits for second order self-adjoint difference equations. J. Math. Anal. Appl. 323, 513-521 (2005)

13. Ricceri, B: A general variational principle and some of its applications. J. Comput. Appl. Math. 133, 401-410 (2000)

14. Bonanno, G, Molica Bisci, G: Infinitely many solutions for a Dirichlet problem involving the $p$-Laplacian. Proc. R. Soc. Edinb., Sect. A 140, 737-752 (2010)

15. Bonanno, G, Candito, P: Infinitely many solutions for a class of discrete non-linear boundary value problems. Appl. Anal. 88, 605-616 (2009)

16. Molica Bisci, G, Repovš, D: On sequences of solutions for discrete anisotropic equations. Expo. Math. 32, 284-295 (2014)

17. Stegliński, R: On sequences of large solutions for discrete anisotropic equations. Electron. J. Qual. Theory Differ. Equ. 2015, 25 (2015)

18. Bonanno, G, Molica Bisci, G: Infinitely many solutions for a boundary value problem with discontinuous nonlinearities. Bound. Value Probl. 2009, Article ID 670675 (2009)

\section{Submit your manuscript to a SpringerOpen ${ }^{\ominus}$ journal and benefit from:}

- Convenient online submission

- Rigorous peer review

Immediate publication on acceptance

- Open access: articles freely available online

- High visibility within the field

- Retaining the copyright to your article 